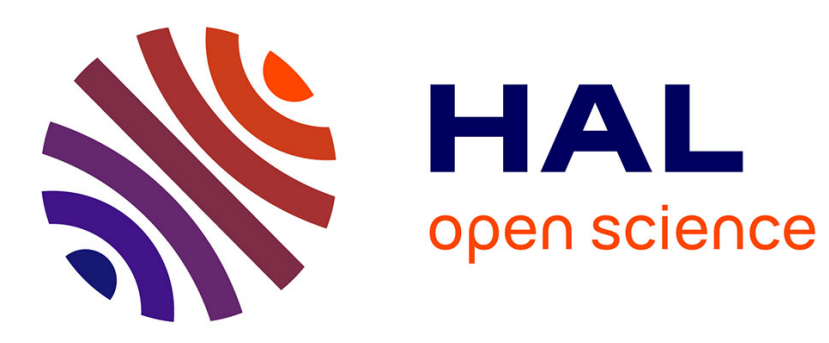

\title{
Tangible Interaction in Mixed Reality Systems
}

Nadine Couture, Guillaume Riviere, Patrick Reuter

\section{To cite this version:}

Nadine Couture, Guillaume Riviere, Patrick Reuter. Tangible Interaction in Mixed Reality Systems. Dubois E., Gray P. and Nigay L. The Engineering of Mixed Reality Systems, Springer-Verlag, pp.101120, 2010, Human-Computer Interaction Series, 978-1-84882-732-5. 10.1007/978-1-84882-733-2_6 . hal-00408042

\section{HAL Id: hal-00408042 \\ https://hal.science/hal-00408042}

Submitted on 15 Dec 2020

HAL is a multi-disciplinary open access archive for the deposit and dissemination of scientific research documents, whether they are published or not. The documents may come from teaching and research institutions in France or abroad, or from public or private research centers.
L'archive ouverte pluridisciplinaire HAL, est destinée au dépôt et à la diffusion de documents scientifiques de niveau recherche, publiés ou non, émanant des établissements d'enseignement et de recherche français ou étrangers, des laboratoires publics ou privés. 


\title{
Tangible Interaction in Mixed Reality Systems
}

\author{
Nadine Couture ${ }^{1,2}$, Guillaume Rivière ${ }^{1,2}$, Patrick Reuter ${ }^{2,3,4}$ \\ ${ }^{1}$ ESTIA-RECHERCHE, Technopole Izarbel, 64210 Bidart, France \\ \{n.couture, g.riviere\}@estia.fr \\ ${ }^{2}$ LaBRI, 351, cours de la Libération, 33405 Talence, France \\ ${ }^{3}$ INRIA Bordeaux - Sud Ouest, 351, cours de la Libération, 33405 Talence France \\ ${ }^{4}$ Université Bordeaux 2, 146 rue Léo Saignat, 33076 Bordeaux, France \\ preuter@labri.fr
}

\begin{abstract}
In this chapter, we discuss the design of tangible interaction techniques for Mixed Reality environments. We begin by recalling some conceptual models of tangible interaction. Then, we propose an engineering-oriented software/hardware co-design process, based on our experience in developing tangible user interfaces. We present three different tangible user interfaces for real-world applications, and analyse the feedback from the user studies that we conducted. In summary, we conclude that, since tangible user interfaces are part of the real world and provide a seamless interaction with virtual words, they are well-adapted to mix together reality and virtuality. Hence, tangible interaction optimizes a users' virtual tasks, especially in manipulating and controlling $3 \mathrm{D}$ digital data in $3 \mathrm{D}$ space.
\end{abstract}

Keywords. Tangible User Interface, Augmented Virtuality, Design Process, Case Studies.

\section{Introduction}

Tangible User Interfaces (TUIs) are one of several genres of sensing-based interaction and they have attracted significant attention during recent years. TUIs were initially defined by [22] as user interfaces that "augment the real physical world by coupling digital information to everyday physical objects and environments". Note, though, that the concept of TUIs is not new and was previously known as Passive Props [19] or as Graspable User Interfaces [14].

To get a taste of tangible interfaces, let us consider two examples. First, Urp (Urban Planning Workbench) [43] allows several users together to control an urban simulation with physical scale models of buildings, manipulating it via a 
physical workbench. The buildings' shadows or wind flow can be simulated and displayed on the workbench, integrated with the scale models. Second, Illuminating Clay [37] allows road builders, environmental engineers, and landscape designers to modify a terrain relief by modeling with their fingers a physical surface that represents the ground. Analytic data, like slope variation, water flow or land erosion can be displayed directly on this surface. Users manipulate the physical terrain model around a workbench and can thus work together in order to design the course of a new roadway, a housing complex or a parking area that satisfies engineering, environmental and aesthetic requirements.

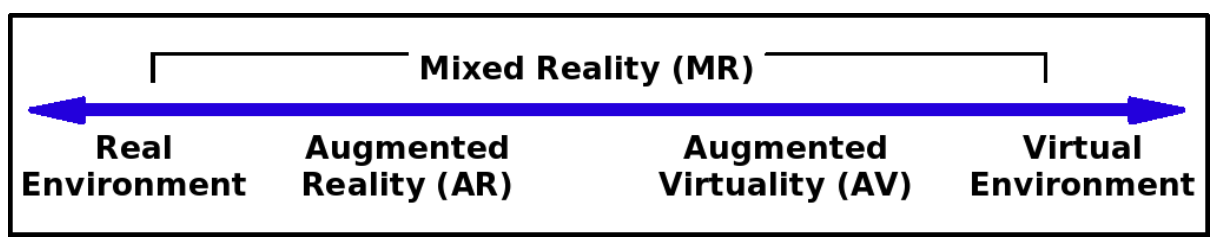

Fig. 1. Reality-Virtuality continuum [30]

We are convinced that real-world physical objects are essential as physical representations and controls of digital information for user interaction. Since TUIs integrate both the physical and digital aspects of interaction, they are as such part of the Mixed Reality (MR) paradigm.

Let us first study the way how the physical and digital world are mixed in a mixed reality system. To this end, consider the "virtuality" continuum, introduced in [30], and illustrated in Figure 1. At one extreme of this continuum, the task is embedded in the physical real-world environment of the users and at the other extreme, the task is embedded in the purely digital virtual environment. Between these extremes, Augmented Reality consists of embedding some digital information in the real-world and Augmented Virtuality consists of embedding some physical information in a virtual world. According to this continuum, in essence, tangible user interfaces are part of Augmented Virtuality. According to [40], tangible interfaces "give physical form to digital information, employing physical artefacts both as representations and controls for computational media”. Recall that in Augmented Virtuality, the task takes place in a virtual environment and the user interacts with this digital information by manipulating physical objects. These physical objects can either represent digital information or the control of digital information in the physical world or, even both, the digital information and its control!

An interactor is the abstraction of an entity capable of representing both input and output [5]. Consequently, a tangible interactor (also called tangible objects, tangibles or props) is a mixed object [8] with a role both in the real-world and the virtual world that are linked by the computer. Tangibles allow the user to perceive and/or to modify the state of the digital information. As an example, in the TUI PinWheels [21] rotations of the pinwheels represent a flow, such as car traffic in a 
street or stock market exchanges. Here, the pinwheels only physically represent the digital information, i.e. a flow, without user control capacity.

Note that a system using physical devices is not necessarily a tangible user interface. Indeed, according to the preceding definitions, "physical" is not a synonym of "tangible". Indeed, work on Graspable User Interfaces already distinguished graspable devices from graspable functions $[12,13]$. The principal characteristic of a TUI is to be a mixed reality system, where the task takes place in the virtual world, which uses augmented virtuality. An interface consisting of physical objects (e.g. mouse, stylus) can neither be simply considered as a TUI, nor as a mixed reality system. A physical object only becomes a tangible object when it represents and/or controls digital information!

This chapter is organized as follows. First, we present a state of the art review of tangible user interface models. Second, taking an engineering approach, we focus on the design of tangible interaction techniques in Mixed Reality environments based on our experiences in designing and building TUIs. Then, we illustrate the previous design steps with three TUI examples that we have developed. Finally, we offer some lessons learnt from the analysis of the feedback from the user studies that we have conducted for each of the TUIs. To conclude, by understanding the world as a 3D environment where TUIs are part of the real world, we show how TUIs are well-adapted to mix together reality and virtuality in order to optimize the users' virtual tasks and to manipulate and control 3D numerical data in 3D space.

\section{State of the art of tangible user interfaces models}

In this section, we initially present the earliest tangible interaction model: the MCRpd [40]. This interaction model is an extension of the classical Model View Control (MVC) principle for graphical user interfaces. Then, we describe the evolution of this initial tangible interaction model to an extended version [20].

\subsection{The seminal tangible interaction model}

The MCRpd model, short for Model-Control-Representation physical and digital, initially introduced by [ $40 ; 41]$ has since 2002 been renamed to MCRit, short for Model-Control-Representation tangible and intangible [39; 42]. 


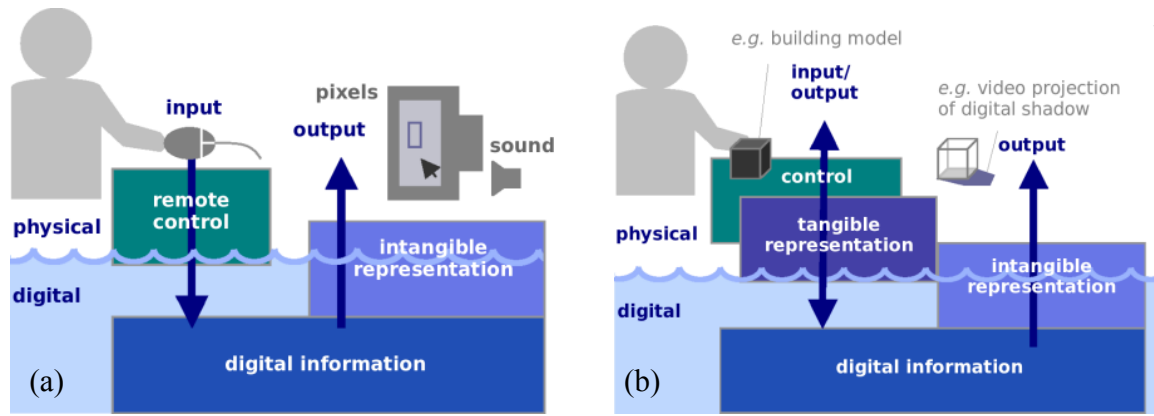

Fig. 2. a. Graphical User Interface Model. b. MCRit, Tangible User Interface Model. (both are reprinted from [20])

By analogy with the MVC model for graphical interfaces, the MCRit model describes the different physical and digital components that occur in a tangible interface. Graphical user interfaces and tangible user interfaces can thus be compared as follows:

- Graphical User Interfaces (GUIs) represent information with intangible pixels on a bit-mapped display and sound. General-purpose input devices allow users to control those representations (see Figure 2-a).

- Tangible User Interfaces (TUIs) make information directly graspable and easily manipulated with haptic feedback, by giving tangible (physical) representation to the digital information. Intangible representation, such as video projection for example, may complement tangible representations by synchronizing with it (see Figure 2-b).

\subsection{The extended tangible interaction model}

In 2008, Ishii [20] introduced an extension to the MCRit model. He states that an interaction with a tangible object is always composed of two feedback loops, and he points out that in some cases even a third feedback loop appears.

The first feedback loop is passive haptic feedback (see Figure 3-a). This feedback loop provides the user with an immediate confirmation that he or she has grasped and moved the physical object. This loop exists within a physical domain, and it does not require any sensing or processing by a computer.

The second loop is digital feedback loop. This feedback loop provides a visual or audible response from the movement of the physical object. Therefore, this second feedback loop implies a computational delay and takes longer than the first loop. 

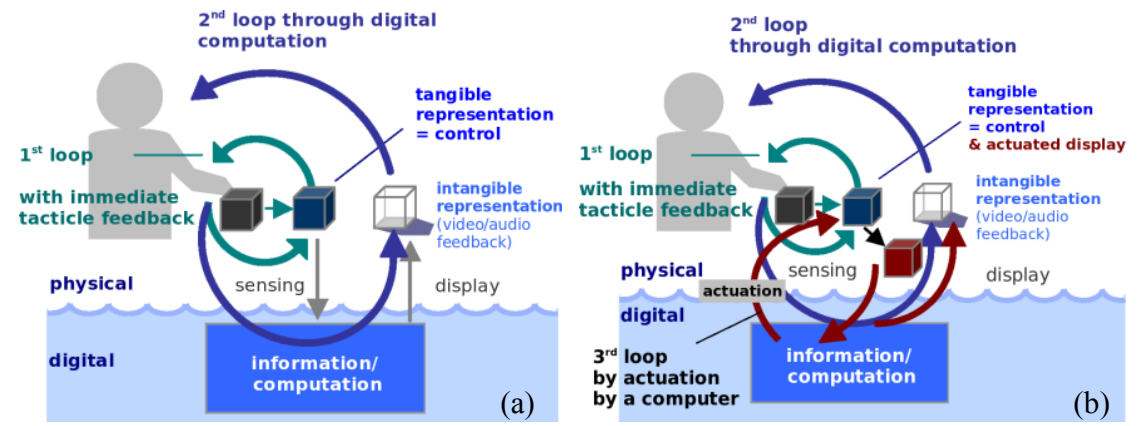

Fig. 3. a. TUI's double feedback Loops. b. TUI with actuation. (both are reprinted from [20])

The third feedback loop, that can be called the physical actuation ${ }^{1}$ loop, can be adjoined to the two preceding loops when the computer gives physical feedback on the status of the digital information as the model changes or responds to internal computation. Hence, the computer generates a physical update (physical actuation) of the tangibles. Figure 3-b illustrates the third loop introduced into the tangible interaction model by computer-controlled actuation and sensing.

As an example, in PICO [34], the pucks move on a tabletop according to the calculations done by an optimization algorithm, so that the user can intervene in computational optimization problems by adding physical constraints on the puck movements (e.g. cell-phone tower placement). Today, in the area of tangible interfaces, there is still a high potential to better exploit the physical actuation loop. The reasons for this are manifold, but they are primarily feasibility (and thus technical) issues. However, there are some promising novel solutions that create physical artefacts that move, animate, or deform themselves: the Actuated Workbench [33], HoverMesh [29], Surflex [6], Sprout I/O [7] and BounceSlider [15].

\section{Designing Tangible Interaction Techniques in MR environments}

The objective in tangible user interface design is twofold. First, the designer has to choose an adequate physical form for representing the digital information and/or the control of the digital information. Second, the designer has to integrate this real product in an interactive system. In order to better understand his or her design, it is useful for the designer to have a categorization of the available system elements. Hence we start this section by outlining two major categorizations of TUIs from the literature.

\footnotetext{
${ }^{1}$ Actuation means "to put in action", "to move".
} 


\subsection{Categorizations of Tangible User Interfaces}

In 2004, Fishkin [11] discussed and analyzed existing TUIs and found no useful binary characteristic function that meaningfully includes some of the TUIs while excluding others. Instead, he proposed a taxonomy that unifies previous and various different definitions and categorizations of TUIs. For that, he found it useful to view "tangibility" as a multi-valued attribute. Fishkin proposed two axes. The metaphor axis classifies the TUI in terms of the way the system effect of a user action is analogous to the real-world effect of similar actions. The embodiment axis classifies the TUI with respect to how closely the input focus is tied to the output focus. Fishkin's taxonomy is the result of unifying into one framework various classifications that existed before 2004 [11].

More recently, in 2008, Ishii [20] introduced an overview of seven types for promising TUI applications based on an analysis of interfaces developed by the research community during the previous ten years: tangible telepresence, tangibles with kinetic memory, constructive assembly, tokens and constraints, tangible interactive surfaces, continuous plastic TUIs, and augmented everyday objects. We refer the interested reader to [20] for details of each type of TUI application and for examples.

\subsection{A multidisciplinary and participatory approach}

We recommend a design methodology that integrates classical methods of both computer science and product design. In our approach, the key element in the early stage of design of a tangible user interface consists of identifying the users' major needs for a new interaction device, taking into account the users' skills and experience in doing the targeted task. We propose that the next stage is multidisciplinary integrating both the product designer and software designer, as well as being a participatory approach that also includes the end user. The goal of this process is to design the right interaction technique and the most suitable device. We should note that while GUIs are fundamentally general purpose interfaces, TUIs are relatively specific interfaces tailored to a certain type of application in order to increase the directness and intuitiveness of the interaction. By taking advantage of existing skills and work practices, the critical task can be identified where TUIs can reveal their best performance. 


\subsection{Taking into account the skills of users}

In order to integrate human factors in the design of the user interface under consideration, it is obviously necessary to take into account the end user of the interface during the design. It is also important to take into account the know-how of the user in order to develop tools that are adapted to the targeted tasks.

An example is ESKUA (described below in section 4.2), a tangible user interface for 3D CAD parts assembly. CAD systems are widely used to design parts and to assemble them, and these systems have become more and more powerful. They now provide very high level functions that allow a user to position many parts with only a few mouse clicks. For example, it is possible to perform a multiple selection on two, three or more objects with one click. Then, bringing their axis in alignment only requires selecting the matching item in a popup menu. Unfortunately, even though these powerful functionalities are very useful from a computer user point of view, they mask real problems that only occur in the final production stage. For example, the operator may not have enough hands to handle all the parts and perform the alignment! Hence, there is obviously a wide gap between the way this action is performed in the CAD system, and the way it is in the real world by taking into account the skills of the operators. As a consequence, we designed ESKUA together with professional CAD users, because the handling of real objects makes it possible to "anticipate" some physical aspects of the product assembly phase, and thus leads the designer to raise questions by carrying out the gestures related to the assembly in the early design stage.

Another example is ArcheoTUI (described below in section 4.3). ArcheoTUI was initiated by the demand of archeologists to improve user interaction for the broken fragments assembly task. We designed ArcheoTUI in a direct collaboration with a team of archeologists, and we proved its efficiency in a case study of the assembly of one of their fractured archeological findings.

\subsection{The design process}

The designer of a tangible user interface first has to design an adequate physical form for representing the digital information and/or the control of the digital information, and second he or she has to integrate this real product into an interactive system. In order to make the design process successful, we propose to undertake a multidisciplinary and participatory approach by implementing a classical engineering design process in 7 steps. At each step, if necessary, the designer can go back to one of the previous steps. The end user is taken into account at every step, and we recommend that the designers should not consider the end user as he or she imagines them, but as he or she actually is! This implies meeting the end users in the early design stage. To this end, we propose the following approach: 
Step 1. Creativity/brainstorming with the end users. Meetings are organized with the end users in order to determine the context of use, the usage scenarios, the data to manipulate, the main tasks and difficulties encountered in the previous way of working. It is important to analyze the end users' spontaneous working conditions. Sometimes, these spontaneous conditions are more likely the way they worked before the arrival of computer systems and the usage of graphical interfaces. At this step of the process, the dialogue between designers and end users makes it possible for the designer to understand the skills of the users. After these first meetings, the designers of the TUIs should present the established requirements list to the end users. Subsequently, meetings should be organized again in order to find the best solution to the problem, where the designers propose various solutions of interfaces and interaction techniques.

Step 2. Intermediate objects/demonstrators. The designers develop initial mock-ups of the solutions that have been adopted and present them to the end users.

Step 3. Adaptation of functional specifications and constraints.

Step 4. Simulations. Note that at this step the first mock-ups do not necessarily use the final technology. Moreover, if the technology is not available, the Wizard of $\mathrm{Oz}$ technique can be used to conduct early user studies (see for example [9]).

Step 5. Setting situations.

Step 6. Development and test. The designers develop a working prototype.

Step 7. User experimentations. First experimental studies are carried out with the end users on the working prototype, in order to validate the design choices.

In the next sections, we validate the design process described above by comparing the designed product/system to the expected product/system. We show a high level of correspondence between them in terms of characteristics, qualities and functionalities. We reinforce the validation of our design approach concretely by the investigations with end users that we conducted in three different applications (see below in Section 5).

\section{Case studies}

By following the design process described above, we designed three TUIs for different fields of use. For each of them, we give a rationale for its creation and illus- 
trate its conception. These three TUIs called ESKUA, GeoTUI and ArcheoTUI, respectively, are case studies of so-called "augmented virtuality" systems.

\subsection{A tangible user interface for 3D CAD parts assembly: ESKUA}

ESKUA is a TUI for interaction with Computer Aided Design (CAD) software. Thanks to its props, ESKUA provides product designers with a physical simulation of parts assembly operations (see Figure 4-a). Each prop is associated with one or more virtual objects, the CAD parts. We have designed and manufactured a set of props made out of 60 elements with holes (see Figure 4-b). The actions that the user carries out on the props (displacement, assembly, rotation, etc.) are reproduced on the CAD parts on the display screen (see Figure 4-c). The capture of the position and the orientation of the props is done by video capture (see Figure 4-d). The use of ESKUA gives the product designer a physical perception of the assembly constraints during the "virtual" CAD part manipulation. Indeed, the designer is confronted with real assembly operation constraints such as difficulties in positioning parts or maintaining elements in a joint position. The props set based on functional surface reasoning makes it possible to carry out physical simulations and allows the designers to identify assembly difficulties and to modify the CAD part design.

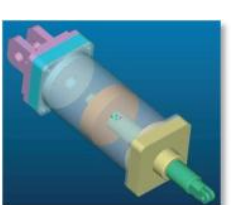

(a)

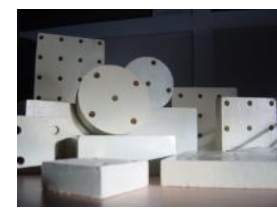

(b)

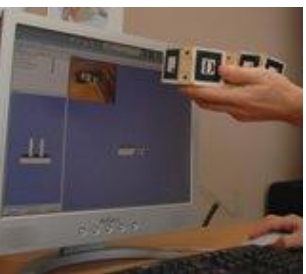

(c)

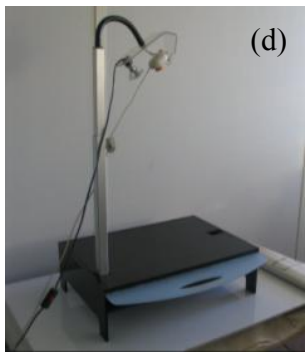

Fig. 4. a. CAO assembly. b. ESKUA tangible props. c. The association of the props to CAD parts. d. The set-up of ESKUA.

Note that, when assembling two elements (CAD parts, archaeological fragments (see ArcheoTUI below), and so on ...), the user has to manipulate double $6 \mathrm{DOF}$ at the same time, and classical user interfaces such as the $2 \mathrm{D}$ mouse or the keyboard are impractical for this assembly task. Using TUIs for assembly is not a new idea. The assembly of numerous Lego-like blocks as props was already done with Active Cubes [25]. Based on the conceptual framework of [17], two-handed manipulation techniques were developed, see for example $[19 ; 28 ; 35]$, and a part of their success can be attributed to their cognitive benefits [26]. Our work is inspired by the seminal work of Hinckley et al. [19] where passive real-world interface props are used for neurosurgical visualization. 


\subsection{A tangible tabletop for Geoscience: GeoTUI}

In the field of energy, a key activity is the search for hydrocarbons by geoscientists. The geophysicists must reconstitute a three-dimensional (3D) model of deep structures by interpreting seismic 3D data (see Figure 5-a) based on their expertise and assisted by powerful geological simulation software.

In order to explore a cubic volume of subsoil, the geophysicists perform vertical cutting planes. Cutting planes are vertical in the cube since it is too difficult for the geophysicists to create a mental 3D representation of the subsoil starting from arbitrarily oriented cutting planes. Before GUIs existed, geophysicists used to cut planes on paper sheets in a non-interactive way. GUIs allow the geophysicists to interactively edit splines and the composition of the subsoil from cutting planes. However, the complexity of the interaction makes the work too difficult for many geophysicists. Moreover, to understand the data, geophysicists often work together with geologists - but sharing a mouse and a keyboard in front of a screen does not necessarily promote such collaboration. Our new tangible user interface (see Figure 5-b) displays cutting planes and geographical maps on a tabletop and provides tangible props for the manipulation of the data. Our aim is to combine the paper/pen/tools conditions of interaction on a table familiar to geophysicists with the computational power of modern geological simulation software. The tangible tools are directly manipulated on intangible cutting planes and maps. According to the recommendations of Norman [31], GeoTUI system has a perfectly coinciding action and perception space. Consequently, the geophysicists concentrate as much as possible on the actual task at hand. Moreover, we strongly believe that tangible interaction for the manipulation of data in the physical world (instead of logical manipulation in the digital world) helps the geophysicists to concentrate more on their actual professional problems.

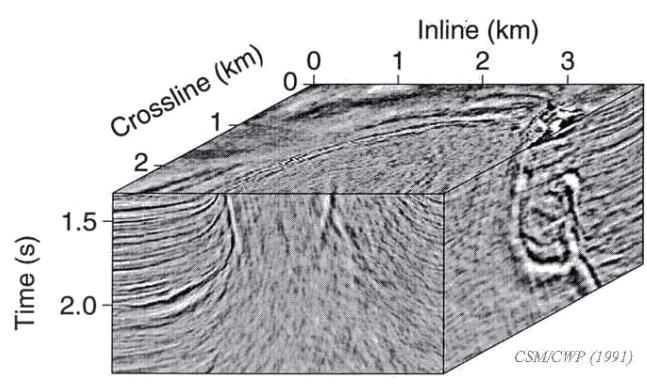

(a)

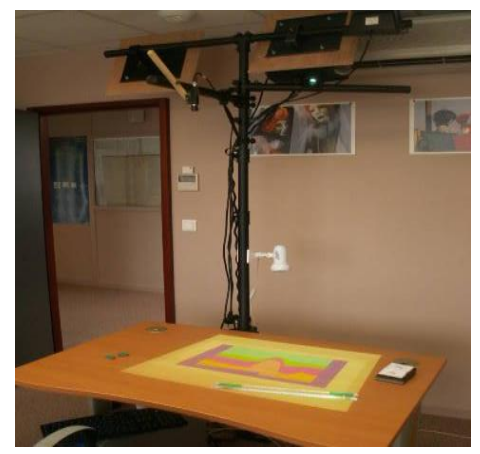

(b)

Fig. 5. a. Seismic 3D volumetric data (CSM/CWP 1991). b. The set-up of GeoTUI.

In order to explore a cube of subsoil and to edit splines and composition, the geologists and geophysicists identify cutting planes. Specifying a cutting line from geographical subsoil maps in order to obtain a cutting plane is a frequent task in 
the geophysicists' work. We focused on this key task in order to develop a prototype and to prove the relevance of tangible tabletop to geoscience. We implemented four means of interaction for navigation in the subsoil model in order to evaluate the best method of interaction for the cutting line selection task. "The best interaction" has to be understood in terms of speed, and, more importantly, in terms of reliability. One is with the mouse on the screen (classical GUI), and three are with tangible props as input, and the tabletop as output: one puck, two pucks and a ruler (see Figure 6-a,b,c). The mouse and the one puck prop are used to repeatedly control the positions of two logical handles of the cutting line that is drawn on the map. The two puck props are two physical handles that allows the geophysicist to control the cutting line that is displayed between them. The graded border of the ruler prop represents the cutting line, and the ruler allows the geophysicist to control the position and the orientation of the cutting line.

The 2D cutting planes cannot be calculated on the fly. In the GUI, a graphical button allows the geophysicists to select the cutting line and to engage the calculation of the $2 \mathrm{D}$ cutting plane. After the calculation, this $2 \mathrm{D}$ cutting plane is displayed instead of the map. In GeoTUI, we propose to couple the use of the props with an additional device: a physical button box (see Figure 6-d). Adding buttons on tangibles is not always a good solution [2;19], so this button box is a solution intended for tangible tabletops. We built this button box consisting of physical buttons in the spirit of Norman [32]. Norman explains the benefits of "physical affordances", not "perceived affordances", and that "people would be better served if we were to return to control through physical objects, to real knobs, sliders, buttons, to simpler, more concrete objects and actions." In the prototype, the button box is composed of four buttons that are labelled exactly the same as the button widgets in the GUI that the geophysicists are used to. When the user validates a cutting line on the map, the cutting plane is displayed instead of the map.
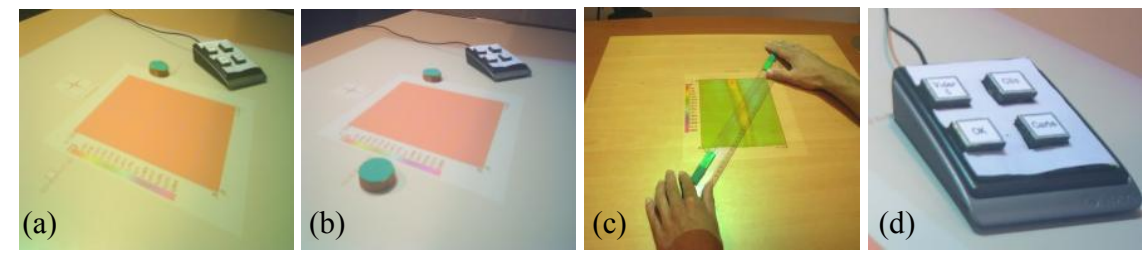

Fig. 6. a. The one-puck prop. b. The two-puck prop. c. The ruler prop. d. The button box.

In the context of a geographical subsoil model, to the best of our knowledge, the GeoTUI system, specifically designed for geophysicists, is the first work that uses tangibles on a tabletop for the specific task of selecting perpendicular cutting planes from a topographic map. It combines the advantages of the spontaneous user interaction that the geophysicists are commonly used to in their classical paper/pen/ruler environment, with the advantages of the use of powerful geological simulation software. 


\subsection{A tangible user interface for the virtual reassembly of fractured archaeological objects: ArcheoTUI}

Objects found during archeological excavations are often broken and fractured into a large number of fragments. A common tedious and time-consuming task for archeologists is to reassemble these fractured objects. Large 3D puzzles have to be solved, so to speak. This task is sometimes made even more difficult because some of the fragments are either very heavy, underwater, deteriorated by erosion and damage, or sometimes even missing.

(a)

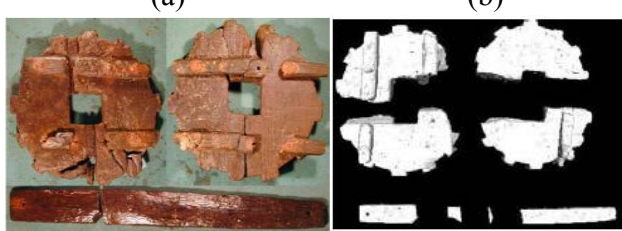

(c)

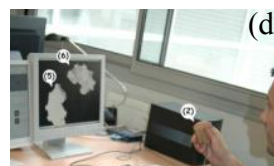

Fig. 7. a. Photos of the fractured fountain parts. b. The virtual fragments. c. The assembly of the virtual fragments. d. The setup of ArcheoTUI.

Various researchers have proposed to scan fragments in 3D in order to use ever-increasing computing power to create a virtual computer-aided assembly (see Figure 7). Once one has figured out how the virtual fragments fit together, the information can be used as a blueprint to reconstruct the real-world object. Even though automatic matching techniques exist, they fail when entire fragments are missing, or when the fragments are seriously deteriorated by, for example, erosion, weathering, or impact damage.

ArcheoTUI is a new tangible user interface for the efficient assembly of 3D scanned fragments of fractured archeological objects. The key idea of the ArcheoTUI system is to use props as physical representation and control for the scanned virtual fragments. In each hand, the user manipulates an electromagnetically tracked prop, and the translations and rotations are directly mapped to the corresponding virtual fragments on the display. For each hand, a corresponding foot pedal is used to clutch the hand movements. Hence, the user's hands can be repositioned or the user can be switched. This declutching mechanism was already used by Hinckley et al. [19] with only one foot pedal, and we extended this metaphor to two foot pedals: the left pedal for the user's left foot is associated with the user's left hand actions and with the right pedal for the right hand's actions, respectively. Foot pedals for two feet were also used by Balakrishnan [2]; however, in contrast to our foot pedals, in their work the role for each foot is not the same. 
The ArcheoTUI software is designed to enable assembly hypotheses to be changed easily, beyond classical undo/redo, since the reassembly of archeological findings is a lengthy trial-and-error task. Once the user has figured out how the virtual fragments fit together, the information can be used as a blueprint to reassemble the real-world archeological object.

\subsection{Illustration of the design approach on case studies}

The three previous examples of tangible interfaces illustrate the design approach presented in Section 3. It is out of the scope of this chapter to detail each step of the design approach for each TUI. Nevertheless, we illustrate the design approach by focusing on the first steps: taking into account the skills of the users (in step 1) and the building of intermediate demonstrators followed by the adaptation of the functional specifications and constraints (step 2 and step 3). Step 7 will be illustrated in Section 5.

Step 1, taking into account the skills of the users, illustrated by ArcheoTUI. The design approach has to be chosen right from the start by taking into account the skills of the users. For example, several meetings with the archeologists convinced us that in archeology, the years of experience of the archeologists is crucial to solving the $3 \mathrm{D}$ assembly puzzle. The archeologists reason not only bottom up by pairwise matching, but also top down, by considering the assembly problem as a whole, and by taking into account the archeological context. We observed that the user interaction techniques involved in classical existing 3D modelling software hinder the efficient virtual assembly of 3D objects, because the two 3D objects have to be positioned and oriented relative to each other. Since the archaeologists are often inexperienced in user interaction with 3D models by using the 2D metaphor of the mouse, in some laboratories, the virtual assembly is slowed down or even completely abandoned. Note that it is already difficult to position and orientate one $3 \mathrm{D}$ object with a 2D metaphor such as the trackball metaphor. Consequently, positioning and orientating two objects relative to each other is even harder, especially for non 3D experts. ArcheoTUI was designed to overcome this difficulty.

Steps 2 and 3, building intermediate objects/demonstrators followed by adaptation of the functional specifications and constraints illustrated by ESKUA. We defined a typology of these tangibles based on concepts proposed in "Design For Assembly" (DFA) methods [4]. Following this first proposal we carried out different investigations [27] to test this first set of tangibles with different types of users: designers, assembly experts, CAD users, and ergonomic experts. Our studies highlight that the subjects use different cognitive techniques to associate the CAD parts with the tangibles. Basically, our experiments show that the subjects 
propose different combinations of tangibles according to two kinds of criteria. The first criterion is the general form of the part (like the DFA principle) and the second one is the functional surface of the part. We then designed a new set of tangibles with specific functional surfaces that are commonly used in the assembly process (such as chamfer on shaft and bore, fillet, flat on shaft, etc.).

\section{User studies in the workplace: feedback}

We provide an analysis of feedback from the user studies that we conducted for each of the previously presented TUIs. For each user study, we present the set-up, the targeted task and the overall success of the interaction technique provided. "Overall success" has to be understood in terms of speed, and, more importantly, reliability. The particular interest of this feedback lies in the fact that the studies were conducted within the user's respective everyday environments - their workplaces.

\subsection{Evaluation: set-up, metrics, analysis}

ESKUA. The principal goal of the user study was to verify the following research questions. Does the use of the props cause a reflection on the assembly? Does the link between the prop and the CAD part depend on the shape or on the functional surfaces? Does the user naturally create new props when the parts have more complex shapes? The subjects were two CAD experts, an assembly expert, an experienced CAD user, and an ergonomist. We provided the subject with a $3 \mathrm{D}$ visualization of a CAD assembly and the set of props. Then, we asked the subject to create the assembly by using the props. This was done for 10 different assembly tasks. In these 10 different assembly tasks, we could qualitatively identify that the subjects have two ways of associating the assembly of the props with the assembly of the CAD parts. The first association is based on reasoning about the geometric shapes, and the second one is based on reasoning about the functional surfaces of the CAD parts. In particular, it appears that the assembly expert, who is the one we address with our research, tries to identify primarily the functional surfaces in order to analyze and optimize the product as a whole.

The main result (see [16] for details) is that the less the users are specialized in the assembly task, the less they reason about the surfaces and the more they reason about the geometries. Therefore, we produced a state of the art report on the technology components that are mainly used in different mechanical products. Furthermore, we proposed a new set of props with specific functional surfaces that are commonly used in the assembly process (e.g. chamfer on shaft and bore, fillet, flat on shaft). Moreover, new "fastening props" had also been designed for the use of 
different fastening technologies (e.g. bolt with nut, screw, centring pin, rivet), and we built additional props in order to promote props combinations regarding both functional surface-based reasoning and geometric form reasoning.

GeoTUI. We conducted two user studies in succession. First, a cognitive walkthrough based user study [36] with 10 participants showed the ability of GeoTUI to support the cutting line selection task. The users were in an exploratory learning mode. The subjects received no instructions about the usage of the two interfaces being compared: the GUI and GeoTUI. The GUI and the GeoTUI interface both controlled the same geoscience software called JOHN [23]. We gave each subject a box containing a ruler, six pucks, and the button box, and we said: "Take them and put them where you want during the exercises [...] Place the button box where it will be most comfortable for you to use." When using GeoTUI, the user had to arrange the objects as he or she liked, and then had to choose the props that he found the most representative for the task of cutting line selection. Second, a formal comparative user study with 12 participants allowed us to evaluate user performance with respect to the usage of the three tangible props: 1-Puck, 2-Puck and the ruler to specify cutting planes. For the two user studies, the order of using the GUI and TUI was counterbalanced, and when testing several props in the second user study, the interaction order was counterbalanced as well within the TUI conditions.

One of the exercises consisted of the selection on the map of a series of six cutting planes at various given coordinates. Another consisted of the selection on the map of cutting planes in order to navigate through a model to find marks hidden in the subsoil at random locations. For the last exercise, the user had to locate and identify a 3D geometric form, shaped as a letter of the alphabet and hidden in a cube, and the user can only view the 2D planes of this cube. All exercises were time-limited.

In the context of geological applications, these experiments allowed us to validate the hypothesis of [12] by convincing quantitative results: the experiment showed that the specialized space-multiplexed conditions outperform the generic space-multiplexed conditions since the task involves a generic working problem that has to be solved by means of the manipulation of input devices (see results and details in [10]). Hence, the ruler is chosen more often as an input device by geophysicists. It may help them to concentrate more on their actual complex working task. Certainly, being able to work in a space where action and perception are unified, thanks to the tabletop, is crucial.

ArcheoTUI. We conducted two user studies on site at the workplace of the archeologists. The subjects had to accomplish a series of 6 assemblies of geometrical shapes or archeological fragments. The first user study revealed that the interface, and especially the foot pedal, was accepted, and that all the users managed to solve simple assembly tasks efficiently (see [38] for details set-up and analysis). 
In a second user study, we replaced the foot pedal declutching mechanism by classical buttons on the props and we compared these two different clutching mechanisms with each other. The conditions were between-subject, and the preference between pedals and buttons was evenly distributed with a slight preference for the foot pedals. This second user study revealed as well that the movement of the hands is more similar to real-world assembly scenarios when using the foot pedals and that the users can keep on concentrating on the actual assembly task.

\subsection{Lessons learnt from the user studies}

The method of evaluation of TUIs compared to GUIs is unusual, since the manipulation of the data happens in the real-world. Based on the instrumental interaction model, Beaudouin-Lafon [3] explains that TUIs "transfer most of the characteristics usually found in the logical part of the instrument into the physical part." The evaluation of a GUI focuses primarily on the logical aspect of the interface. For GUIs, the set of physical actions is limited (see the UAN notation [18]). For TUIs, the physical part, i.e. the aggregation of several tangible objects, is developed specifically for each interface. Furthermore, the physical manipulation does not deal with the graspable input devices, but with physical representations of digital information. Some TUIs are especially designed to exploit bi-manual interaction, but users often perform two handed manipulations with TUIs even if it was not especially designed with that in mind. Having multiple, tangible objects encourages two handed interaction [12], and experiments should examine how users take advantage of it, or if it is sometimes cumbersome to use [24]. The previous analyses allow us to draw some recommendations in order to evaluate TUIs.

Recommendations derived from our user studies on tangible interaction. During our evaluation, some information was gathered by simple observation, e.g., in the particular case of interactive tangible tabletops, the locations of the tangible objects on the table, as well as the way the user arranges and organizes his or her workspace. By studying the position of the hands of the user on the tangible object, one can discriminate between a single handed or bimanual interaction, with one or two tangibles. It is also interesting to study how the tangible object is held, for example grasped with the whole hand or only with the fingertips, in the middle or at the ends. The position of the user, e.g. sitting, standing, or alternating between both, provides interesting information about eventual problems concerning accessibility particularly in the case of interactive tables.

For the comparison of two tangible interactors, the respective manipulation times between two delimiting actions can be analyzed. For example, for GeoTUI, in order to measure the time needed to specify a cutting line, we measured the time difference between the visualization and the validation action. As another example, for ArcheoTUI, we measured the time difference between the clutching 
and declutching of a fragment to the props, both for the foot pedal mechanism and the buttons on the props.

The $2 \mathrm{M}$ conception space [1] analyses the bimanual interaction according to three axes: the nature of the action (discrete, continuous, or composed), the temporality (anachronistic, sequential, concomitant, coinciding, or simultaneous), and the dependence (lexical fusion, syntactic, semantic, or independence otherwise). This theoretical basis allowed us to analyze the TUIs that use bimanual interaction. For example, for ArcheoTUI, we have analyzed the temporality of the two symmetric and continuous bimanual actions with two independent subtasks. We wanted to determine whether the user really interacts with the two props at the same time, and whether one prop was used more frequently than another. It is useful from a design point of view to study the chronology of every single action of the user and to calculate the interlacing of two continuous actions by distinguishing the situations where a unique action is realized from the situations where both actions are done at the same time.

In summary, the most important fact is that physical manipulation is the salient point to be measured during the evaluation.

To this end, in order to collect all this information, we recall some useful techniques: an observer can fill out a form during the experiment or after the experiment by analyzing a video of the experiment, and a log file can be used as well. Moreover, in order to collect a sufficient amount of data to assess the proposed tangible interaction, we recommend conducting two types of investigation successively. Firstly, a cognitive walkthrough-based user study compares the major choices and prepares the second and more precise evaluation. Secondly, a comparative user study evaluates the user performance.

Some questions as a guide. The user study protocol has to be constructive with the aim of establishing the relevant criteria for the major points described above. In order to help the evaluator to evaluate the tangible system and to define the criteria, we propose some questions as a guide. By answering these questions, the evaluator follows the previous recommendations.

Q1- Are the tangible props representative of the role they play?

Q2- Can the tangible props be easily seized with the hands?

Q3- Are the tangible props manipulated by one or two hands or even in cooperation by various users at a time?

Q4- Are the tangible props manipulated sequentially or in parallel?

Q5- Does the manipulation of the tangible props influence the actual task?

Q6- Is the use of the tangible props with the digital world intuitive?

Q7- Is the use of the tangible props efficient?

There is no order to these questions; rather, they should be considered in parallel. The first five require simple yes/no answers, whereas the last two questions require some qualitative judgement. It is often useful to fill out an observation form. 
The user study protocol has to be set up so that the answers to these questions can be clearly identified, with sufficient statistical evidence.

\section{Conclusion: the benefits of tangible interaction in mixed reality systems}

When considering the world as a 3D environment, TUIs are part of the real world, and they interact with digital information. We presented a conceptual analysis of TUI models in order to understand tangible interaction. It is easy to see that TUIs are well-adapted to mixing reality and virtuality together in a mixed reality system, especially when they provide two-handed 3D interaction. As we saw in the case studies, the users interact with mixed reality systems by means of TUIs with a minimum of cognitive workload. This is because TUIs provide a seamless interaction with virtual worlds.

Based on the user studies we have conducted, we have provided an analysis of the feedback, and we have offered some recommendations for the evaluation of TUIs. The key point is to measure the physical manipulation during the evaluation. We presented seven questions that we consider useful in order guide the designer in evaluating a tangible system. They deal with the use of tangibles, manual manipulation, the sequential/parallel way of using tangibles, and the affordance of the tangible. In order to collect a sufficient amount of data for the assessment of the proposed tangible interaction and to answer the seven questions, we recommend conducting two forms of evaluation: a cognitive walkthrough-based user study, followed by a comparative user study for a more precise evaluation of user performance.

Moreover, based on our experience in building tangible interfaces for different fields of use, we presented a hardware/software co-design for tangible user interfaces. Tangible user interfaces are leaving the conventional computer-generated virtual world behind, moving into the physical world. Obviously, the designer of a TUI has to conceive both the physical part and the logical part of the interface. For this reason, we recommend using a classical engineering design process for mechanical products with an end user participatory approach coupled with a software development method. This results in a multidisciplinary approach straight from the beginning of the conception of TUIs by integrating the end user, the product designer, and the software designer.

This design process could help the designer to choose an adequate physical form for representing the digital information and/or the control of the digital information. This design process helps to integrate the tangible object into an interactive system as well. The salient point is, from our perspective, to take into account the users' skills and users' experience in performing the task in question. 


\section{References}

1. Bailly, G., Nigay, L., Auber, D.: 2M: un espace de conception pour l'interaction bimanuelle. In: UbiMob'05 the 2nd French-speaking conference on Mobility and ubiquitous computing, pp. 177--184. ACM (2005)

2. Balakrishnan, R., Fitzmaurice, G., Kurtenbach, G., Singh, K.: Exploring interactive curve and surface manipulation using a bend and twist sensitive input strip. In I3D '99 the 1999 symposium on Interactive 3D graphics, pp. 111--118. ACM (1999)

3. Beaudoin-Lafon, M.: Instrumental Interaction: An Interaction Model for Designing Post-WIMP User Interfaces. In: CHI'00 the 18th SIGCHI conference on Human factors in computing systems, pp. 446--453. ACM (2000)

4. Boothroyd, G., Dewhurst, P.: Product Design for Assembly - A Designer's Handbook. Departement of Mechanical Engineering, University of Massachusetts (1993)

5. Calvary, G., Daassi, O., Coutaz, J., Demeure, A.: Des widgets aux comets pour la Plasticité des Systèmes Interactifs. J. RIHM Revue d'Interaction Homme-Machine, 6, 1, 33$-53(2005)$

6. Coelho, M., Ishii, H., Maes, P.: Surflex: a programmable surface for the design of tangible interfaces. In: CHI'08 extended abstracts of the 26th SIGCHI conference on Human factors in computing systems, pp. 3429--3434. ACM (2008)

7. Coelho, M., Maes, P.: Sprout I/O: A Texturally Rich Interface. In: TEI'08 the 2nd international conference on Tangible and embedded interaction, pp. 221--222. ACM (2008)

8. Coutrix, C., Nigay, L.: Mixed Reality: A model of Mixed Interaction. In: AVI'06 the 8th International Working Conference on Advanced Visual Interfaces, pp. 43--50. ACM (2006)

9. Couture, N., Minel, S.: TactiMod dirige et oriente un piéton. In: UbiMob '06 the 3rd French-speaking conference on Mobility and Ubiquitous computing, pp. 9--16. ACM (2006)

10. Couture, N., Rivière, G., Reuter, P.: GeoTUI: a tangible user interface for geoscience. In: TEI'08 the $2^{\text {nd }}$ international conference on Tangible and Embedded Interaction, $p$. 89--96. ACM (2008)

11. Fishkin, K.: A taxonomy for and analysis of tangible interfaces. J. PUC Personal and Ubiquitous Computing. 8, 5, 347--358 (2004)

12. Fitzmaurice, G.: Graspable User Interfaces. PhD thesis, University of Toronto (1996)

13. Fitzmaurice, G., Buxton, W.: An empirical evaluation of graspable user interfaces: Towards specialized space-multiplexed input. In: CHI'97 the 15th SIGCHI conference on Human factors in computing systems, pp. 43--50. ACM Press (1997)

14. Fitzmaurice, G., Ishii, H., Buxton, W.: Bricks: laying the foundations for graspable user interfaces. In: CHI'95 the 13th SIGCHI conference on Human factors in computing systems, pp. 442--449. ACM Press/Addison-Wesley Publishing Co (1995)

15. Gabriel, R., Sandsjö, J., Shahrokni, A., Fjeld, M.: BounceSlider: actuated sliders for music performance and composition. In: TEI'08 the 2nd international conference on Tangible and embedded interaction, pp. 127--130. ACM (2008) 
16. Garreau, L., Legardeur, J., Rouillon-Couture, N.: Une plate-forme basée sur les interfaces tangibles pour l'assemblage en CFAO. J. Ingénierie Numérique, Interaction Homme-Machine et CAO. 1, 2, pp.133-148. Lavoisier-Hermes (2005)

17. Guiard, Y.: Asymmetric division of labor in human skilled bimanual action: The kinematic chain as a model. J. Motor Behavior. 19, 486--517 (1987)

18. Hartson, H.R., Siochi, A.C., Hix, D.: The UAN: a user-oriented representation for direct manipulation interface designs. J. TOIS the ACM Transactions on Information Systems. 8, 3, 181--203 (1990)

19. Hinckley, K., Pausch, R., Goble, J., Kassell, N.: Passive Real-World Interface Props for Neurosurgical Visualization. In: CHI'94 the 12th SIGCHI conference on Human factors in computing systems, pp. 452--458. ACM (1994)

20. Ishii, H.: Tangible Bits: Beyond Pixels. In: TEI'08 the 2nd International Conference on Tangible and Embedded Interaction, pp. XV--XXV. ACM Press (2008)

21. Ishii, H., Ren, S., Frei, P.: Pinwheels: visualizing information flow in an architectural space. In: CHI'01 extended abstracts of the 19th SIGCHI conference Human factors in computing systems, pp. 111--112. ACM (2001)

22. Ishii, H., Ullmer, B.: Tangible bits: towards seamless interfaces between people, bits and atoms. In: CHI'97 the 15th SIGCHI conference on Human factors in computing systems, pp. 234--241. ACM (1997)

23. Jurado, F., Sinoquet, D., Lailly, P.: Jerry: a 3D reflection tomography designed for complex structures. Technical report, KIM 1996 Annual Report, Institut Français du Pétrole (1996)

24. Kabbash, P., Buxton, W., Sellen, A.: Two-handed input in a compound task. In: CHI '94 the 12th SIGCHI conference on Human factors in computing systems, pp. 417--423. ACM (1994)

25. Kitamura, Y., Itoh, Y., Kishino, F.: ActiveCube: A Bi-directional User Interface using Cubes. In: CHI'01 extended abstracts of the 19th SIGCHI conference on Human factors in computing systems, pp. 355--356. ACM (2001)

26. Leganchuk, A., Zhai, S., Buxton, W.: Manual and cognitive benefits of two-handed input: an experimental study. J. TOCHI. 5, 4, 326--359 (1998)

27. Legardeur, J., Garreau, L., Couture, N.: Experiments to evolve toward a tangible user interface for CAD parts assembly. In: SPIE'04 Electronic Imaging'04, pp. 438--445. SPIE (2004)

28. Llamas, I., Kim, B., Gargus, J., Rossignac, J,. Shaw, C.: Twister: a space-warp operator for the two-handed editing of 3D shapes. In: SIGGRAPH '03: ACM SIGGRAPH 2003 Papers, pp. 663--668. ACM (2003)

29. Mazzone, A., Spagno, C., Kunz, A.: The HoverMesh: a deformable structure based on vacuum cells: new advances in the research of tangible user interfaces. In: ACE'04 the 2004 ACM SIGCHI International Conference on Advances in computer entertainment technology, pp. 187--193. ACM (2004)

30. Milgram, P., Kishino, F.: A Taxonomy of Mixed Reality Visual Displays. J. IEICE Transactions on Information Systems, E77-D, 12, 1321--1329 (1994)

31. Norman, D.: The Psychology of Everyday Things. Basic Books, New York (1988)

32. Norman, D.: Affordance, conventions, and design. J. Interactions. 6, 3, 38--43 (1999)

33. Pangaro, G., Maynes-Aminzade, D., Ishii, H.: The Actuated Workbench: computercontrolled actuation in tabletop tangible interfaces. In: UIST'02 the 15th annual ACM symposium on User Interface Software and Technology, pp. 181--190. ACM (2002). 
34. Patten, J., Ishii, H.: Mechanical constraints as computational constraints in tabletop tangible interfaces. In: CHI'07 the 25th SIGCHI conference on Human factors in computing systems, pp. 809--818. ACM (2007)

35. Pierce, J., Stearns, B., Pausch, R.: Voodoo dolls: seamless interaction at multiple scales in virtual environments. In: I3D '99 the 1999 symposium on Interactive 3D graphics, pp. 141--145. ACM (1999)

36. Polson, P.G., Lewis, C., Rieman, J., Wharton, C.: Cognitive walkthroughs: a method for theory-based evaluation of user interfaces. J. Man-Mach. Stud. 36, 5, 741--773 (1992)

37. Piper, B., Ratti, C., Ishii, H.: Illuminating Clay: a 3-D tangible interface for landscape analysis. In: CHI'02 the 20th SIGCHI conference on Human factors in computing systems, pp. 355--362. ACM (2002)

38. Reuter, P., Rivière, G., Couture, N., Sorraing, N., Espinasse, L., Vergnieux, R.: ArcheoTUI - A Tangible User Interface for the Virtual Reassembly of Fractured Archeological Objects. In: VAST2007 the 8th EuroGraphics International Symposium on Virtual Reality, Archaeology and Cultural Heritage, pp. 15--22. EuroGraphics Association (2007)

39. Ullmer, B.: Tangible Interfaces for Manipulating Aggregates of Digital Information. $\mathrm{PhD}$ thesis, Massachussetts Institute of Technology (2002)

40. Ullmer, B., Ishii, H.: Emerging frameworks for tangible user interfaces. J. IBM System Journal, 39, 3/4, 915--931 (2000)

41. Ullmer, B., Ishii, H.: Emerging frameworks for tangible user interfaces In: HumanComputer Interaction in the New Millenium, pp. 579--601. ACM Press/AddisonWesley Publishing Co (2001)

42. Ullmer, B., Ishii, H., Jacob, R.: Token+constraint systems for tangible interaction with digital information. J. TOCHI 12, 1, 81--118 (2005)

43. Underkoffler, J., Ishii, H.: Urp: a luminous-tangible workbench for urban planning and design. In: CHI'99 the 17th SIGCHI conference on Human factors in computing systems, pp. 386--393. ACM (1999) 